\title{
Johann Jakob Scheuchzer im europäischen Geistesleben seiner Zeit
}

Es gibt, namentlich in der Biologie, wissenschaftliche Probleme, deren Lösung sich nicht durch eine einmalige Anstrengung eines Einzelnen oder einer einzigen Generation von Forschern erzwingen ließ, sondern die sich durch die Jahrhunderte hinziehen. So reicht zum Beispiel die Beschäftigung mit der Frage, ob es eine Urzeugung gebe, das heißt, ob unter gewissen Bedingungen kleinste Lebewesen, ohne daß Keime vorhanden sind, fortgesetzt neu entstehen können, von Aristoteles bis Pasteur. $\mathrm{Zu}$ diesen Problemen gehört auch die Frage nach der Natur der Versteinerungen, der Fossilien, denn ihr Studium reicht von der Frühzeit griechischer Forschung, etwa vom Jahre 600 vor Christus, bis tief ins 18. Jahrhundert. In einem bewegten Moment dieser Forschung ist der Zürcher Johann Jakob Scheuchzer hervorgetreten. ${ }^{1}$

Es sei mir gestattet, selbst auf die Gefahr hin, vielen von Ihnen Wohlbekanntes vorzubringen, mit einigen Worten auf die Anschauungen des Altertums über die Natur der Versteinerungen einzutreten.

Die alten Griechen kannten das Meer und die Tiere des Meeres. Deshalb vermochten sie, wenn Überreste von Meertieren weitab vom Meere auf dem Festlande gefunden wurden, diese Überreste als solche von marinen Tieren zu erkennen. Sie zogen aus diesen Beobachtungen den Schluß, daß die Gegenden, in denen sich versteinerte Überreste von Meertieren finden, einst vom Meere bedeckt gewesen sein müßten. Die griechi-

Aulavortrag, gehalten am 16. November 1944. Für die Aufnahme dieser Studie im «Gesnerus» bin ich dem Herausgeber sehr zu Dank verpflichtet. Die Wiedergabe entspricht dem mündlichen Vortrag; lediglich der Schlußpassus über Malta, für den beim Vortrage die Zeit nicht ausreichte, ist hinzugefügt worden.

1 Die beste Wegleitung durch Scheuchzer's Werk ist das «Verzeichnis des wissenschaft. lichen Nachlasses von Johann Jakob Scheuchzer», das Bibliothekar Dr. Rudolf Steiger im Auftrag der Zentralbibliothek Zürich herausgegeben hat und das in der Vierteljahrsschrift der Naturforschenden Gesellschaft in Zürich in Band LXXVIII, 1933 erschien. Dieses Verzeichnis enthält neben einer vollständigen, chronologisch geordneten Aufführung der gedruckten Publikationen eine nach Materien geordnete Übersicht über den sehr umfangreichen handschriftlichen Nachlaß und über die Korrespondenz, die nicht weniger als 57 Manuskriptbände umfaßt. Dieser Übersicht ist ein alphabetisch geordnetes Verzeichnis der Korrespondenten beigefügt. Von der Scheuchzer-Biographie, die Rud. Steiger begonnen bat, ist bisher erst der Anfang, «Werdezeit» überschrieben, 1926 als Dissertation in Zürich erschienen. In dieser Arbeit wird auck auf die früheren Scheuchzer-Biographien, nament. lich auf diejenige von Rudolf Wolf (1858) verwiesen. 
schen Fachschriften über Versteinerungen, namentlich eine solche von Theophrast, dem großen Schüler des Aristoteles, sind verloren gegangen; ${ }^{2}$ doch haben die Erkenntnisse griechischer Wissenschaft einen Niederschlag in der römischen Dichtung hinterlassen. Berühmt ist eine Stelle in den Metamorphosen, in denen $\mathrm{Ovid}^{3}$ den Pythagoras gleichsam als einen antiken Ahasver sagen läßt:

Vidi ego quod fuerat quondam solidissima tellus, Esse fretum: vidi factas ex aequore terras;

Et procul a pelago conchae jacuere marinae, Et vetus inventa est in montibus ancora summis:

Ich sah zum Meer geworden, was einst festeste Erde war. Ich sah aus dem Meer entstandene Länder.

Fern vom Meere lagen marine Muscheln, und uralt ist der Anker, Im Gipfel des Berges gefunden.

Die Nennung der Conchae marinae, der Meermuscheln, beruht auf Beobachtung; den Anker hat Ovid aus dichterischer Machtvollkommenheit von sich aus hinzugetan, denn die Alten hatten noch keine Vorstellung von der ungeheuren Größe der Zeiträume, welche die Veränderungen in der Gestalt der Erdoberfläche und in der Verteilung von Land und Wasser erforderten.

Das Mittelalter, das auf den Untergang der römischen Kultur folgte, war für die Entwicklung der Naturwissenschaften wenig günstig. Die alten Erkenntnisse waren in Vergessenheit geraten und von abenteuerlichen Vorstellungen überwuchert worden. Insbesondere wurden die Versteinerungen als Naturspiele, als lusus naturae, erklärt, deren Bildung man sich mit ungezügelter Phantasie in der verschiedensten Weise ausmalte. ${ }^{4}$ So standen die Dinge zu Ende des Mittelalters. Ein geographisches Moment mag zu dieser ungünstigen Entwicklung beigetragen haben, denn wenn nunmehr beim Bergbau oder beim Steinbruchbetriebe, viele Hunderte von Kilometern vom Meere entfernt, Versteinerungen zum Vor-

2 Hinsichtlich der Ansichten der alten Griechen und der Römer über die Versteinerungen sei auf Karl A. von Zittel's «Geschichte der Geologie und Paläontologie bis Ende des 19. Jahrhunderts», Band 23 der «Geschichte der Wissenschaften in Deutschland», München und Leipzig 1899, verwiesen.

3 Ovid, «Metamorphosen», Buch XV, Vers 262-265.

${ }_{4}$ Auch hinsichtlich der Meinungen des Mittelalters über die Versteinerungen, sei auf die in Fußnote 2 genannte Darstellung von Zittel verwiesen. 
schein kamen, die dazu oft durch Schichtdruck deformiert waren, so war es für einen Beobachter, der meist das Meer selber nie gesehen hatte, kaum möglich, in diesen Resten marine Organismen zu erkennen. Bezeichnenderweise trat nun die Meinung, die Fossilien seien Überreste von Tieren, die einst wirklich gelebt hätten, zuerst wieder in Italien auf, das heißt in einem Lande mit ausgedehnter Meeresküste und dazu mit zahlreichen Vorkommen von jungtertiären Ablagerungen mit eindrucksvollen, wohlerhaltenen Fossilien, die den Vergleich mit rezenten Objekten nahelegten. Der große Leonardo da Vinci ${ }^{5}$ war der erste, der auf Grund eigener Beobachtungen die wahre Natur der Fossilien mit Sicherheit wieder erkannte, allein seine trefflichen Feststellungen und seine scharfsinnigen Schlußfolgerungen wirkten sich nicht aus, sondern sie blieben, in Spiegelschrift aufgezeichnet, in seinen Tagebüchern vergraben. Ähnliche Anschauungen vertraten in der Folge der um die Förderung der Keramik hochverdiente Bernard Palissy ${ }^{6}$ in Paris, Fabio Colonna ${ }^{7}$ in Rom und andere. Etwa 150 Jahre nach Leonardo, im Jahre 1669, erschien die entscheidende Arbeit des großen dänischen Forschers Nicolaus Steno, ${ }^{8}$ der damals in Florenz lebte. Steno war von Haus aus Arzt. Anatomische Studien an Haifischen hatten ihn dazu geführt, die sogenannten Glossopetren, die Zungensteine, näher zu untersuchen und in ihnen mit Sicherheit Zähne von Haifischen zu erkennen. Die Untersuchung ausdehnend, suchte er die Unterschiede zwischen organischen Resten und Kristallen zu erfassen und fand dabei für die Kristalle das Gesetz der Winkelkonstanz der

5 Leonardo da Vinci wurde bei der Gelegenheit eines Kanalbaues in Oberitalien, dessen Leitung er inne hatte, auf Fossilien aufmerksam.

6 Bernard Palissy, «Discours admirables de la nature des eaux et fontaines, des métaux, des sels et salines, des pierres, des terres, du feu et des émaux etc.», Paris 1580. Noch 1768 versuchte Voltaire die Ideen von Palissy über die Natur der Fossilien lächerlich zu machen; das XVII. Kapitel von Voltaire's «Des singularités de la nature» ist betitelt: «Idées de Palissy sur les coquilles prétendues».

7 Fabio Colonna. «Osservazioni sugli animali aquatici e terrestri», Roma 1616.

8 Nicolaus Steno, dessen anatomische Untersuchungen zu Entdeckungen von größter Tragweite geführt haben, lebte von 1638 bis 1686. Der Titel der im Jahre 1669 erschienenen grundlegenden Arbeit lautet «De solido intra solidum naturaliter contento». Ins Deutsche wurde diese Arbeit von Karl Mieleitner übertragen. Diese Übersetzung ist in Ostwald's Klassikern der exakten Wissenschaften 1923 erschienen. Mit Unterstützung durch den Carlsbergfonds konnte Vilhelm Maar unter dem Titel: «Nicolai Stenonis Opera philosophica» Steno's wissenschaftliche Arbeiten in gediegener Weise herausgegeben. Das in Kopenhagen 1910 erschienene Werk wird durch eine Lebensbeschreibung und eine Würdigung der Arbeiten Steno's eingeleitet. 
begrenzenden Flächen. Beim Studium des Schichtenbaues erkannte er, $\mathrm{da}$ ß fossilführende Schichten in geneigter oder senkrechter Lage diese Position erst durch sekundäre Vorgänge erreicht haben können und wurde dadurch zum Begründer der Tektonik und schließlich zählt er durch den Versuch, eine geologische Geschichte der Toscana zu entwerfen, zu den Begründern der Stratigraphie.

Man darf sich aber nun nicht vorstellen, daß mit dem Erscheinen von Steno's Arbeit die neue Lehre sich rasch und allgemein durchgesetzt hätte. Im Gegenteil! Durchmustert man die Jahrgänge der ersten Jahrzehnte der Ephemerides der Leopoldina, ${ }^{9}$ der Zeitschrift der deutschen Akademie, die 1670, also ein Jahr nach Steno's Arbeit zu erscheinen hegann, nach Arbeiten über Fossilien, so sieht man erst, wie lange es ging, bis die neuen Erkenntnisse sich ganz allmählig durchsetzten.

$\mathrm{Zu}$ den Anhängern des Alten gehörten auch Schweizer, unter anderen der Zürcher Arzt Johann Jakob Wagner, ${ }^{10}$ der in zweierlei Hinsicht Johann Jakob Scheuchzer's Vorgänger war, einmal in der beruflichen Stellung als Waisenhausarzt und sodann als Verfasser einer Naturgeschichte des Schweizerlandes. In dieser 1680 erschienenen Naturgeschichte preist Wagner noch die therapeutischen Qualitäten von pulverisierten Belemniten und in einer 1685 in den Ephemerides erschienenen Observatio wendet er sich gegen die Torheit derjenigen, die in den Zungensteinen Zähne von Haifischen erblicken wollten.

Johann Jakob Scheuchzer selber - er ist 1672 in Zürich geboren hat, als er 1692-1694, an der Universität Altorf bei Nürnberg mehr Naturwissenschaften als Medizin studierte, dort mit Eifer Versteinerun-

9 Der Titel der noch heute existierenden Zeitschrift, der im Laufe der Zeit mehrmals geändert wurde, lautete für den ersten Band: «Miscellanea curiosa medico-physica Academiae naturae curiosorum sive ephemeridum medico-physicarum germanicarum curiosorum annus primus.» Über die Frühgeschichte der Leopoldinischen Akademie orientiert Andreas Elias Büchner's «Academiae S.R. J. Leopoldino-Carolinae naturae curiosorum Historia.» Halle 1755.

10 Johann Jakob Wagner lebte von 1641 bis 1695. Über sein Verhältnis zu Scheuchzer siehe R. Steiger's Scheuchzer-Biographie, pag. 24 und 102. Wagner's «Historia naturalis helvetiae curiosa» ist in paläontologischer Hinsicht durch Ortsangaben von Fossilfunden woch heute von Interesse. Die erwähnte Observatio 189 im dritten Jahrgang der zweiten Decuria der Ephemerides ist betitelt: «Lapilli albi Caryophyllos aromaticos referentes». Die vermeintlichen Gewürznelken sind, wie aus der leider ohne Abbildung veröffentlichten Beschreibung mit Sicherheit hervorgeht, in Wirklichkeit die zierlichen kleinen Kelche der fossilen Seelilie Eugeniacrinus caryophyllatus. 
gen gesammelt. Er hielt aber damals die Muschelsteine, die Conchiten, noch für anorganische Produkte, deren Bildungsweise er auf physikalischchemischem Wege zu erklären versuchte. Eine Publikation darüber erschien in den Ephemerides des Jahres 1697.11 Scheuchzer setzte sich dabei auch mit Steno auseinander, dem er immerhin die Konzession macht, daß die Conchiten gewisser Schichten Uberreste von marinen Muscheln darstellen könnten. In seiner trefflichen Scheuchzer-Biographie hat Rudolf Steiger geschildert, wie Scheuchzer nach seiner Rückkehr nach Zürich im «Collegium der Wohlgesinnten» über das gleiche Thema Vorträge hielt, deren Manuskript noch vorliegt.

Der radikale Umschwung in Scheuchzer's Anschauungen erfolgte unter dem Eindruck des Werkes eines Engländers, John Woodward, das 1695 in London erschienen war. ${ }^{12}$ Scheuchzer hat diese Arbeit übersetzt, nicht etwa ins Deutsche, sondern, um ihr im ganzen gelehrten Europa Verbreitung zu verschaffen, ins Lateinische. Woodward anerkannte die Fossilien als Überreste von Organismen, die einst wirklich gelebt haben. Die Ursache der großen Katastrophe, die all diese Lebewesen vernichtete, erblickte er in der Sintflut. ${ }^{13}$ Diese Lehre fand, weil sie sich auf ihre Ubereinstimmung mit dem Berichte der Genesis berufen konnte, großen Beifall. Ihre Vertreter nannte man die Diluvianer. Scheuchzer ist nicht der Begründer dieser Lehre, aber er wurde ihr erfolgreichster Vertreter dadurch, daß er sich planmäßig eine große Fossiliensammlung anlegte and in ungezählten Einzelfällen immer wieder auf die Übereinstimmung der Fossilien mit rezenten Pflanzen- und Tierresten hinwies.

Verglichen mit den Erkenntnissen, zu denen Steno vorgedrungen war, bedeutete die Sintflutlehre einen Rückschritt, einen Fortschritt dagegen gegenüber den alten Anschauungen, die in den Versteinerungen immer noch nichts als Figurensteine und Naturspiele erblicken wollten. Ein Schweizer Vertreter der alten Richtung war der Luzerner Ratsherr Carl

11 Die Arbeit findet sich im Appendix zu Jahr V und VI der dritten Decuria der Ephemerides, der 1700 in Nürnberg erschien. Sie ist in Form eines Briefes an Michael Friedrich Lochner abgefaßt unter dem Titel: «Dissertatio epistolica Acarnanis de Dendritis sliisque Lapidibus, qui in superficie sua plantarum, foliorum, florum figuras exprimunt.» 12 John Woodward (1665-1722), «Essay towards a natural history of the earth and terrestrial bodies etc. Der Titel von Scheuchzer's lateinischer Übersetzung dieses Werkes lautet: «Specimen geographiae physicae qua agitur de terra et corporibus terrestribus etc.»

${ }_{13} \mathrm{Im}$ 《Antlitz der Erde» (Dritte Auflage, Wien 1908, pag. 25-53) hat Eduard Sueß den biblischen Bericht über die Sintflut, sowie die babylonischen Texte vom geologischen Standpunkte aus eingehend diskutiert. 
Nicolaus Lang. ${ }^{14}$ Seine an sich gründliche «Historia lapidum figuratorum Helvetiae» ist 1708 erschienen, im gleichen Jahre, in dem als Arbeit aus dem Lager der Diluvianer Scheuchzer's «Piscium querelae et vindiciae», die bewegten Klagen der Fische, erschienen. Die deutsche Ausgabe trägt den Titel: «Bildnussen verschiedener Fischen, und dero Theilen, welche in der Sündflut zu grund gegangen».

Ein frommer Sinn, Ehrfurcht vor den Werken des Schöpfers spricht aus allen Arbeiten Scheuchzer's. Den machtvollsten Ausdruck fand diese Gesinnung in dem Riesenwerk der Physica sacra, ${ }^{15}$ in dem Scheuchzer in vier Foliobänden mit gegen 800 Kupfertafeln dem Leser im Rahmen eines Bibelwerkes und in engem Anschluß an Schriftstellen naturwissenschaftlichen Bildungsinhalt vermittelt. Psalmstellen geben ihm z. B. den Anlaß, unter Benützung der besten damaligen Originalarbeiten, den Bau der Sinnesorgane darzustellen. Für die Tafel «Das Ohr, ein Werk Gottes» ist z. B. die hervorragende Arbeit von Joseph Duverney ${ }^{16}$ benützí. Natürlich ließ sich Scheuchzer die Gelegenheit nicht entgehen, unter dem Kapitel Sündflut die ganze Versteinerungskunde mit einem gewaltigen Aufwand an trefflichen Abbildungen abzuhandeln.

Wie Scheuchzer, waren nicht wenige der Forscher aus dem 17. und dem Beginn des 18. Jahrhunderts tief religiös. Man denke nur an Blaise Pascal, an Nicolaus Steno, der eine glänzende wissenschaftliche Laufbahn aufgab, um sich in Not und Armut ganz dem religiösen Leben zu widmen, und an den Anatomen J. Winslow, der, wie Steno, aus innerem Drang zum Katholizismus übertrat. In den Skulpturen, die das Portal so manchen mittelalterlichen Kirchenbaues, wie z. B. des Berner Münsters, zieren, gelangen auch die räumlichen Vorstellungen des Mittelalters über die Lokalisation des Himmels mit seinen Engeln zu sinnenfälligem Ausdruck. Der Einbruch in diese Vorstellungswelt, der durch das kopernikanische Weltsystem erfolgte, war tiefgreifend. Es ist nun bedeutsam, daß die Naturforschung zu Ende des 17. und zu Anfang des 18. Jahrhunderts, wie sie durch Scheuchzer in typischer Weise verkörpert wird, selber an mathematischen Fragen lebhaft interessiert, zur Verbrei-

14 Carl Nicolaus Lang lebte von 1670 bis 1741.

15 «Physica sacra Johannis Jacobi Scheuchzeri», Augsburg 1731-1735. Die holländische Übersetzung trägt den treffenden Titel: «Geestelyke natuurkunde». Um die Herausgabe dieses Riesenwerkes hat sich der unternehmende Johann Andreas Pfeffel verdient gemacht.

16 Guichard Joseph Duverney (1648-1730). «Traité de l'organe de l'ouie contenant la structure, les usages et les maladies de toutes les parties de l'oreille». Paris 1683. 
tung der neuen Erkenntnisse beitrug, sich aber durch die Veränderung des Weltbildes nicht von ihrer tief religiösen Grundhaltung abbringen ließ.

Das Bestreben, die Forschungsresultate mit dem Wortlaut der heiligen Schrift in Einklang zu bringen, war nicht durch äußeren Druck erzwungen, sondern es kam von innen heraus. Auf geologisch-paläontologischem Gebiete brachte die enge Schriftverbundenheit einerseits ein mächtiges Aufblühen der in dieser Aufmachung von der Geistlichkeit nicht nur tolerierten, sondern begünstigten Forschung; andererseits aber traten mit der Vermehrung des Tatsachenmateriales und mit der Vertiefung der Reflexion Schwierigkeiten zu Tage, die dazu führten, daß die Sintfluthypothese allmählig von besser begründeten Vorstellungen über das Geschehen in der erdgeschichtlichen Vergangenheit abgelöst wurde.

Scheuchzer hatte geglaubt, auch Überreste von Menschen gefunden zu haben, die in der Sintflut zugrunde gegangen sein sollten, nicht nur den bekannten Homo diluvii testis aus Oehningen, ${ }^{17}$ in dem später Georges Cuvier das Skelett eines fossilen Riesensalamanders erkannte, sondern auch weitere Überreste, die sich als Ichthyosaurierwirbel erwiesen haben. Diese Wirbel beschrieb Scheuchzer 1708. ${ }^{18}$ Im gleichen Jahre äußerte Johann Jakob Baier, der nachmalige Präsident der Leopoldina, in einem Briefe an Scheuchzer seine Zweifel. Er erklärte die betreffenden Wirbel für Ichthyospondyliten, für Fischwirbel, eine für den damaligen Stand der Kenntnisse nicht schlechte Diagnose. Dieser Brief wurde 22 Jahre später, drei Jahre vor Scheuchzer's frühem Hinschied, 1730, in den Ephemerides veröffentlicht. $\mathrm{Ob}$ wohl damit eine Opposition eingeleitet werden sollte ${ }^{19}$ Es ist denkbar, daß es, wenn Scheuchzer länger gelebt

17 Homo diluvii testis et $\Theta \varepsilon \delta ́ \sigma \varkappa o \pi \circ \varsigma$, Zürich 1726.

18 Piscium querelae et vindiciae, Zürich 1708, pag. 22; «vertebrae binae dorsale.s humanae petrefactae ex agro Altorfino, Territorii Norimbergensis».

19 Der äußere Anlaß zu der späten Veröffentlichung des Briefwechsels zwischen Baier und Scheuchzer ergab sich daraus, daß Baier ein Supplementum zu seiner Oryctographia norica herausgab. Daß sowohl Scheuchzer wie Baier Ichthyosaurus-Wirbel vor sich hatten, ist zweifellos. Baier schreibt von «seinen» Spondyli: «Hos quidem non omisi conferre cum humanis, sed ab iisdem non parum differre observavi. Praeterquam enim, quod sinus illius, quem foramen commune pro transitu spinalis medullae efficit, nulla appareant in Tuis pariter ac meis spondylis vestigia, profundior cavitas in singulis, acetabuli adinstar, et eam congrue explens corpus, in centro notabiliter acuminatum, quicquam aliud quam humanae vertebrae conditionem naturalem prodit.» Es ist schade, daß Scheuchzer bei seiner 
hätte, schon damals zu einer genaueren Untersuchung des Homo diluvii testis gekommen wäre, bei der Scheuchzer selber die Unhaltbarkeit seiner Deutung der fraglichen Reste wohl hätte anerkennen müssen. Der speziellen Lehre der Diluvianer hätte der Ausfall dieses vermeintlichen Beweisstückes wohl Eintrag getan, aber die Auffassung der Fossilien als wirklicher Überreste von einstigen Lebewesen hätte er nicht mehr zu erschüttern vermocht.

Viele Jahrzehnte nach Scheuchzer's Tod nahm Voltaire Stellung zum Fossilienproblem. Er wendete sich mit beißendem Hohn gegen alle die Beobachter, die tief im Binnenlande und namentlich in bedeutender Höhe über dem Meeresniveau Überreste von marinen Organismen festgestellt zu haben glaubten und daraus auf eine einstige Meeresbedeckung geschlossen hatten. Noch im Jahre 1768 schrieb Voltaire: ${ }^{20}$

«Tous ces petits fragments de coquilles, dont on a fait tant de bruit pour accréditer un système, sont pour la plupart si informes, si usés, si méconnaissables, qu'on pourrait également parier que ce sont des débris d'écrevisses ou de crocodiles, ou des ongles d'autres animaux. Si on trouve une coquille bien conservée dans le cabinet d'un curieux, on ne sait d'où elle vient; et je doute qu'elle puisse servir de fondement à un système de l'univers.»

Die weitere Entwicklung hat Voltaire nicht Recht gegeben, sondern die von ihm verhöhnten und ins Lächerliche gezogenen Beobachtungen wurden zu Bausteinen einer Erdgeschichte. Es bleibt aber immerhin bemerkenswert, daß er nicht nur polemisierte, sondern sich materiell zu informieren suchte. So sagt er über das Fehlen von marinen Muscheln auf Bergeshöhen:

"Il n'y en a pas une seule sur la châne des hautes montagnes, depuis la Sierra-Morena jusqu'à la dernière cime de l'Apennin. J'en ai fait chercher sur le mont Saint-Gothard, sur le Saint-Bernard, dans les montagnes de la Tarentaise: on n'en a pas découvert.»

späteren Veröffentlichung über den Homo diluvii testis die Einwände Baier's aus dem Jahre 1708 nicht genügend berücksichtigte.

20 «Des singularités de la nature, par un académicien de Londres, de Bologne, de Péterbourg, de Berlin, etc.»; hier zitiert nach der Ausgabe von M. Beuchot, Tome XLIV, (Mélanges, Tome VIII), Paris 1831. Scheuchzer wird von Voltaire nicht erwähnt; Voltaire's Ausfälle richten sich hauptsächlich gegen De Maillet's «Telliamed ou Entretiens d'un Philosophe indien avec un Missionnaire françois.» 
Wenden wir uns nun einem anderen Gebiete zu, auf dem Scheuchzer's Forschung in ganz Europa Beachtung und Anerkennung fand. Schon bei seinem Studienaufenthalt in Deutschland und in Holland war Scheuchzer oft Fragen nach der Natur seiner Heimat, insbesondere nach den Schweizeralpen begegnet. Er machte es nun nicht so, wie noch vor kurzem jener Spaßvogel, der seinen gutgläubigen Hörern allerlei Bären aufband, wie gleich an der Grenze das Herdengeläute beginne und wie sein Vater hinter dem Hause einen privaten Gletscher zum Kühlen des Bieres besitze, sondern Scheuchzer fand, daß er selber über die Alpen im Grunde herzlich wenig wisse. So zog er nach seiner Rückkehr nach Zürich Jahr für Jahr zur Forschung in die Alpen aus. Drei dieser Alpen-

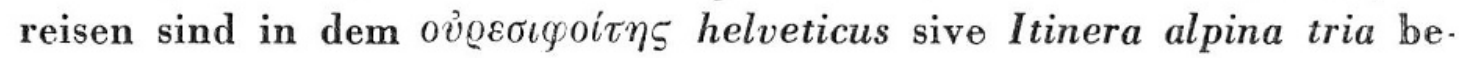
schrieben. ${ }^{21}$ Dieser helvetische Alpenwanderer ist 1708 in London erschienen, offenbar unter persönlicher Beteiligung einer Anzahl von Mitgliedern der Royal Society, der Scheuchzer selber angehörte, denn auf jeder der zahlreichen Kupfertafeln ist der Name eines englischen Stifters vermerkt. Aus dem vielgestaltigen Inhalt seien namentlich die barometrischen Höhenmessungen hervorgehoben; auf einer der Tafeln ist Scheuchzer's Reisebarometer abgebildet. Resultate von barometrischen Beobachtungen hat Scheuchzer auch mehrfach in den Philosophical Transactions veröffentlicht. ${ }^{22}$ Eine eingehende Beschreibung des Bades Pfäfers kam den lebhaften balneologischen Interessen der damaligen Medizin entgegen. Sodann finden sich neben zahlreichen botanischen, zoologischen und mineralogischen Angaben auch volkskundlich wertvolle Schilderungen. Sehr anziehend ist die genaue Beschreibung all der Geräte, die in der alpinen Milchwirtschaft Verwendung finden, wobei sich mitten in dem lateinischen Text die Angabe der lokalen schweizerdeutschen Bezeichnungen, wie z. B. «Stunckenwerne» (item ein feistes Mues, q. d. jusculum pingue, conficitur ex Cremore Lactis et farina invicem coctis, additis insuper, si ad manus fuerint, ovis), und die "Seumelkteren» (vasis immundi genus, Tab. X, Fig. U.; Infimum quidem obtinet in Casis

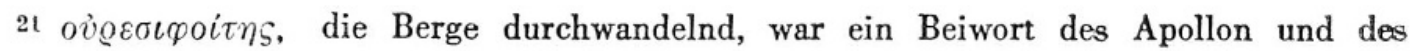
Dionysos. Eine ähnliche Wortbildung findet sich im Neu-Griechischen, indem in dieser

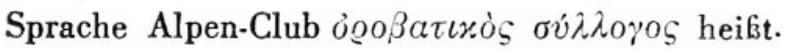

22 «Tables of the barometrical altitudes, Zürich 1708, observed by J. J. Sch., and at Upminster obs. at the same time by W. Derham.» Philos. Transact. 26 (London 1710). «The barometrical method of measuring the height of mountains», Philos. Transact. 35 (London 1729). 
Vaccariis locum hoc Instrumentum, sed primum, ubi de Hospitibus commode locandis sermo fit, scandula rudi tectum. Es ist diß der vornemste Sitz bey den Sennen, oder in Sennhütten) sehr drollig ausnimmt. Scheuchzer hat für die naturwissenschaftliche Erschließung der Alpen zu einer Zeit, als noch außerordentlich wenig bekannt war, durch eigene Initiative sehr viel geleistet. Sein Interesse galt aber nicht nur den Alpen, sondern dem ganzen Schweizerlande. 1699 versandte er einen lateinisch und deutsch gedruckten Einladungsbrief zur «Erforschung natürlicher Wunderen, so sich im Schweizerland befinden». Auf Grund der Vorarbeiten erschienen dann die Beschreibung der Natur-Geschichten des Schweizerlandes, sowie die Behandlung einzelner Gebiete, wie der Hydrographie und der Meteorologie.

Die internationale Bedeutung Scheuchzer's auf den genannten Gebieten dürfte darin liegen, daß er mit hinreißender Begeisterung und mit unermüdlichem Arbeitseifer am Beispiel der Schweiz gezeigt hat, was für die Erforschung eines Landes getan werden kann. Sein Streben ging dahin, die Schweiz zu einem der bestuntersuchten Länder zu machen.

Was Scheuchzer an medizinischen Beobachtungen, namentlich in den Ephemerides, ${ }^{23}$ veröffentlicht hat, scheint nicht über das Mittelmaß hinauszugehen. Er betätigte sich auch als Numismatiker, sowie auf dem Gebiete der Geschichte und ihrer Hilfswissenschaften. Eine von ihm herrührende Sammlung von Urkundenkopien ist dadurch von Bedeutung geworden, daß in manchen Fällen die Originale inzwischen verlorengegangen sind. Auf diesen Teil von Scheuchzer's Werk sei hier nicht eingegangen, weil er mehr lokalen Charakter trägt. Immerhin kamen auch diese Gebiete in der gewaltigen Korrespondenz zur Sprache, die er mit Gelehrten in ganz Europa unterhielt. Viele der Briefe galten seiner Fossiliensammlung. ${ }^{24}$ Von dieser Sammlung hat sich ein bedeutender Teil erhalten. Er ist, da es sich zum größten Teil um fossile Tierreste handelt, von Kollegen Däniker in dankenswerter Weise dem Zoologischen Museum zur Bearbeitung überwiesen worden. Es liegt nun die

23 Acta physico-medica Acad. nat. cur. II, Nürnberg 1730, enthält zehn medizinische Observationes von Johann Jakab Scheuchzer.

24 Scheuchzer hat die fossilen Pflanzen und pflanzenähnliche Bildungen seiner Sammlung 1709 und 1723 unter dem Titel «Herbarium diluvianum» beschrieben und großenteils abgebildet. Die ganze Sammlung, namentlich die fossilen Überreste von Tieren, beschrieb er 1716 als «Museum diluvianum». Über die erhaltenen Bestände wird eine besondere Mitteilung in der Vierteljahrsschrift der Naturforschenden Gesellschaft in Zürich erfolgen. 
Aufgabe vor uns, an Hand von Korrespondenz und Sammlungsobjekten im Einzelnen zu verfolgen, wie Johann Jakob Scheuchzer der hervorragendste «Diluvianer» geworden ist.

Zum Schluß sei mir gestattet, an einem Beispiel zu zeigen, wie es nun möglich ist, in den erhaltenen Beständen der Scheuchzer'schen Sammlung Originale zu Abbildungen in Scheuchzer's Werken festzustellen und so aus dem Vergleich von Objekt und Abbildung wertvolle Einblicke zu erhalten. Auf Tafel 736 der Physica sacra bildet Scheuchzer die Szene ab, die im Schlußkapitel der Apostelgeschichte erzählt wird. Das Schiff, das den Apostel Paulus nach Rom führte, hatte vor Malta Schiffbruch erlitten.

«Und da wir auskamen, erfuhren wir, daß die Insel Melite hieß. Die Leutlein aber erzeigten uns nicht geringe Freundschaft, zündeten ein Feuer an, und nahmen uns alle auf, um des Regens, der über uns gekommen war, und um der Kälte willen. Da aber Paulus einen Haufen Reiser zusammenraffte und legte es aufs Feuer, kam eine Otter von der Hitze und fuhr Paulo an seine Hand. Da aber die Leutlein sahen das Tier an seiner Hand hangen, sprachen sie untereinander: Dieser Mensch muß ein Mörder sein, welchen die Rache nicht leben läßt, ob er gleich dem Meer entgangen ist. Er aber schlenkerte das Tier ins Feuer, und ihm widerfuhr nichts Übels. Sie aber warteten, wenn er schwellen würde, oder tot niederfallen. Da sie aber lange warteten und sahen, daß ihm nichts Ungeheures widerfuhr, verwandten sie sich und sprachen, er wäre ein Gott.»

Diese Erzählung wurde zum Anlaß dafür, daß die Einwohner von Malta im 17. Jahrhundert den Reisenden, die dort ankehrten, Schlangenaugen - in Wirklichkeit waren es fossile Zähne der Dorade, eines noch heute im Mittelmeergebiet hochgeschätzten Speisefisches - als Amulette gegen Vergiftungen aller Art verkauften, dazu zum gleichen $Z_{w e c k}$ auch fossile Haifischzähne. In der kunstvollen Umrahmung, die in Scheuchzer's Tafel die figürliche Darstellung umgibt, ist ein solcher Haifischzahn abgebildet, und dazu ein schlangenförmig gewundenes Gehäuse des marinen Röhrenwurmes Serpula, aus dem ein zierliches Köpfchen hervorschaut. Scheuchzer bemerkt im Text, daß die Malteser, um die Schlangenähnlichkeit vollkommener zu machen, diese Köpfchen aus Lehm formten und in die Serpularöhren hineinsteckten. Das Exemplar von Serpula, wie auch der Haifischzahn, die Scheuchzer abbildete, sind in der Sammlung noch vorhanden. Wenige Jahrzehnte vor Scheuchzer hatte ein Dr. Tude- 
cius de Monte Galea ${ }^{25}$ aus Prag den Schlangenaugen eine Arbeit gewidmet und ihre Heilwirkung gegen Vergiftungen angepriesen. Scheuchzer wußte mit diesen Resten besseres anzufangen; er hat der Erkenntnis zum endgültigen Durchbruch verholfen, daß die Fossilien Überreste von Tieren sind, die einst wirklich gelebt haben.

B. Peyer.

\section{Schweizerische Bibliographie der Geschidhte der Naturwissenschaften und der Medizin. I938 - 1943, III. Serie}

\section{Neuere Paracelsus-Literatur*}

Achelis, J. D. Der Arzt Paracelsus. Die Pause, 6. Jahrgang, 9. April, Wien 1941, (illustr.).

Acta Paracelsica. Im Auftrag der Paracelsus-Gesellschaft herausgegeben von Darmstädter, Koch \& Schröter. Heft 1-5, München 1930-1932.

Albrecht, Alice Sus. Theophrastus Paracelsus. Von seiner Auffassung über Mensch und Natur. Gesundheit und Wohlfahrt, Zürich, Juli 1941.

Allendy, R. Le médecin maudit. Gallimard, Paris 1937.

- - Paracelso, il medico maledetto. Fr. Bocca, Milano 1942.

Artelt, Walter. Paracelsus im Urteil der Medinzinhistorik. Fortschr. d. Medizin 50, Nr. 22, (1932).

- - Paracelsus und seine Zeit in Zeittafeln. "Theophrastus Paracelsus» von Jaeger 1941, vide Jaeger.

Bayon, H. P. Paracelsus: personality, doctrines and his alleged influence in the reform of medicine. Proceedings of the Royal Society of Medicine, London, 35, 69-76, Section of the History of Medicien 9-16. (1941).

Bazala, Vlad. Paracelsus in Kroatien. Neue Ordnung, Zagreb, 21. Sept. 1941.

25 Ephemerides der Leopoldina, Annus IX/X der ersten Decuria, Observatio 119; \&De oculis serpentum et linguis Melitensibus».

* Da es zur Zeit unmöglich ist, die internationale Paracelsus-Literatur auch nur mit annähernder Vollständigkeit zu registrieren, wurde die wichtigere Paracelsus-Literatur bis 1930 zurück verfolgt. Für weitere bibliographische Angaben bin ich den Lesern dankbar. Bis 1930 wurde die Paracelsus-Literatur durch K. Sudhoff in «Nachweise zur ParacelsusLiteratur» (Beilage zu den Acta Paracelsica 1-5, 1930-1932) in 1089 Nummern zusammengestellt.

Eine größere Zahl mir unbekannter Literaturzitate verdanke ich der Güte von P. Dr. Ildefons Betschart in Einsiedeln, welcher in den Nova Acta Paracelsica Bd. I. (Birkhäuser Basel 1944) ebenfalls eine durch gegenseitige Ergänzung konforme Paracelsusbibliographie erscheinen läßt. 